\title{
NOUVELLe
}

\section{Un candidat vaccin lentiviral anti-Covid-19 administrable par voie intranasale}

Laleh Majlessi, Pierre Charneau

\author{
Laboratoire Commun Pasteur-TheraVectys, \\ Institut Pasteur, \\ 28 rue du Docteur Roux, 75015 Paris, France. \\ laleh.majlessi@pasteur.fr \\ pierre.charneau@pasteur.fr
}

> Une immunité collective contre le virus SARS-CoV-2 (severe acute respiratory syndrome-coronavirus 2), un $\beta$-coronavirus responsable d'un syndrome respiratoire aigu sévère, s'installe progressivement dans les populations humaines à la suite des vagues épidémiques de la Covid-19 (coronavirus disease 2019) qui se succèdent depuis la fin de l'année 2019, et grâce à l'utilisation massive des vaccins de première génération dirigés contre la glycoprotéine des spicules de l'enveloppe de ce virus, la protéine Spike. Des arguments expérimentaux ou cliniques indiquent cependant que cette immunité pourrait être d'assez courte durée. II est également à craindre qu'elle soit peu efficace contre certains nouveaux variants du virus qui émergent actuellement, ou qui émergeront si la circulation du virus se prolonge. Dans ce contexte, des vaccins de seconde génération seront nécessaires pour renforcer l'immunité collective contre ce virus [1,2]. Nous avons tiré profit des propriétés uniques des vecteurs lentiviraux de transfert de gènes pour développer un candidat vaccin prophylactique contre la COVID-19. Ces vecteurs ont une grande efficacité de transfert, y compris pour des transgènes de grande taille. Les particules virales sont généralement pseudotypées avec la glycoprotéine d'enveloppe du virus de la stomatite vésiculaire, un virus infectant principalement le bétail et auquel les populations humaines ont été très peu exposées. Par conséquent, contrairement aux vecteurs adénoviraux, aucune pré-immunité contre ce vecteur ne vient en contrecarrer l'efficacité lors de la vaccination. La préimmunité anti-vecteur est un problème majeur en vaccinologie car elle peut réduire l'efficacité du vecteur en inhibant le transfert du gène d'intérêt, en éliminant trop rapidement les cellules transduites, et en formant des complexes immuns qui peuvent être très réactogènes [3]. Les vecteurs lentiviraux à usage vaccinal ont un tropisme préférentiel pour les cellules dendritiques immatures, qui sont au centre de la mise en place de l'immunité adaptative. Cette programmation génétique des cellules dendritiques par les vecteurs lentiviraux permet une présentation de l'antigène efficace par la voie endogène, une propriété unique à ces vecteurs, qui se traduit par l'induction d'une immunité adaptative forte et durable, non seulement de type humoral, mais aussi à médiation cellulaire, avec en particulier la contribution de lymphocytes $T \mathrm{CD}^{+}$, comme cela a été montré dans de nombreux modèles pré-cliniques [3, 4]. À l'opposé des vecteurs lentiviraux intégratifs utilisés pour la thérapie génique et pour la production des lymphocytes $T$ porteurs d'un récepteur de l'antigène chimérique (chimeric antigen receptor $T$ cells, ou cellules (AR-T) à des fins d'immunothérapie, ceux adaptés à la vaccination sont non-intégratifs, ce qui élimine le risque de génotoxicité. L’ADN des vecteurs lentiviraux non-intégratifs reste dans le noyau des cellules dendritiques sous forme d'épisomes circulaires. Les vecteurs lentiviraux sont non-réplicatifs, non-cytopathiques et non-réactogéniques ${ }^{1}$, et leur innocuité a été montrée,

\footnotetext{
1 Réactogénicité : propriété d'un vaccin de produire des réactions indésirables sous forme d'une réponse immunologique excessive se manifestant par de la fièvre, de la douleur à l'endroit de l'injection accompagnée d'un gonflement, une induration et une rougeur attendus, car déjà identifiés et caractérisés dans les essais cliniques.
}

notamment par un essai clinique de phase I d'immunothérapie contre l'infection par le virus VIH-l (virus de l'immunodéficience humaine de type 1) (2011-006260-52 EN). Parmi les nombreuses applications des vecteurs lentiviraux à usage vaccinal, on peut citer l'induction d'une immunité stérilisante contre le virus Zika. Cette immunité est très rapidement et durablement établie dans un modèle murin après une seule injection d'un vecteur lentiviral codant la protéine d'enveloppe $\varepsilon$ de ce virus [5]. Nous avons également montré, chez la souris, que le vecteur lentiviral a une efficacité supérieure à celle du vecteur adénoviral de sérotype 5 dans l'induction des lymphocytes $\mathrm{T} C D 8^{+}$présentant des spécificités multiples et de type mémoire, ainsi que dans l'immunothérapie anti-tumorale [6]. Afin de développer un candidat vaccin contre la Covid-19, nous avons produit un vecteur lentiviral codant la séquence peptidique complète de la glycoprotéine Spike du SARS-CoV-2 (LV::S) [7]. Les coronavirus sont caractérisés par cette glycoprotéine de fusion, ancrée dans leur enveloppe et qui, sous sa forme homo-trimérique, interagit avec le récepteur cellulaire ACE2 (angiotensin-converting enzyme 2), une exopeptidase présente à la surface d'une grande variété de cellules humaines [8]. Le monomère de Spike possède un ectodomaine constitué des sous-unités S1 et $\mathrm{S} 2$, un domaine transmembranaire et un court segment interne. La sous-unité Sl abrite le domaine RBD (receptor-binding domain), le domaine de liaison au récepteur ACE2. Ce domaine contient de nombreux épitopes conformationnels, cibles principales d'anticorps neutralisants qui empêchent l'entrée du virus dans les cellules hôtes [8]. 


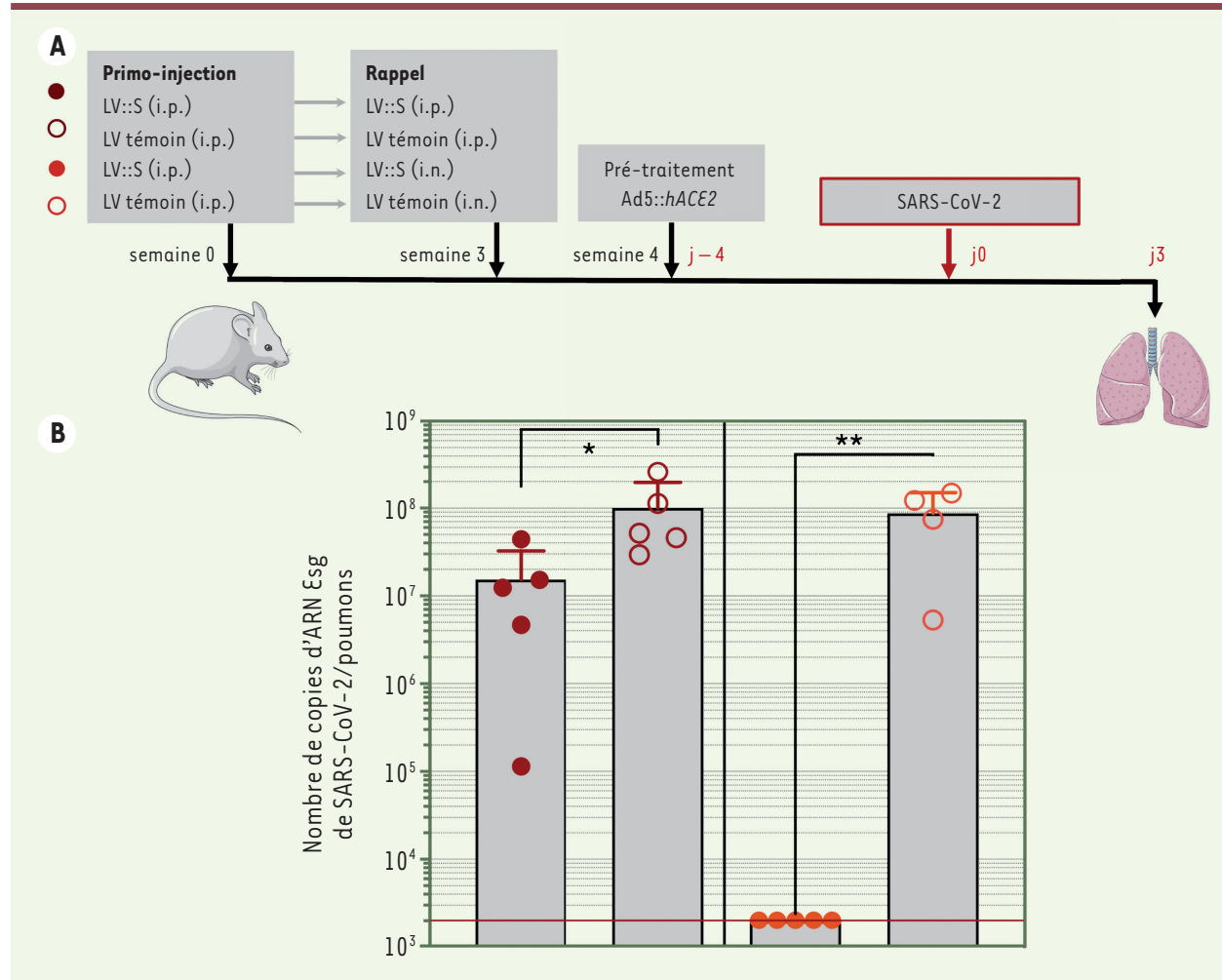

Figure 1. Une injection de rappel du vecteur lentiviral recombinant LV::S par voie intranasale protège entièrement contre le virus SARS-CoV-2 dans un modèle murin de l'infection. (A) Protocole de vaccination de souris $\mathrm{C} 57 \mathrm{BL} / 6$ par $\mathrm{LV}:: S$, suivie d'un prétraitement par le vecteur Ad5::hACE2 codant le récepteur ACE2 humain, et de l'administration du SARS-CoV-2 par voie intranasale 4 jours plus tard. i.p. : intrapéritonéal ; i.n. : intranasal. Le vecteur LV témoin code un antigène non pertinent pour l'infection. Le code couleur des différents groupes d'animaux testés ( 5 animaux par groupe) est indiqué à gauche de cette figure. (B) Charges virales pulmonaires déterminées au $3^{\mathrm{e}}$ jour (j3) après l'inoculation du virus ( $j 0)$, par une technique de RT-qPCR spécifique d'un ARN subgénomique ( $\varepsilon s g)$ exprimé par le virus en cours de réplication. La ligne rouge indique le seuil de détection de l'essai. (c) Titres d'IgG ou d'IgA dirigées contre la protéine spike du SARS-CoV-2 (antiSARS-CoV-2), déterminés par la technique ELISA, dans les homogénats pulmonaires. (D)

Activité neutralisante des homogénats pulmonaires sur l'infection par le virus. La signification statistique des différences observées a été évaluée par le test $U$ de Mann-Whitney $(*: p<0,02 ; * \star: p<0,01)$. Figure adaptée de [7].

Le LV::S, lorsqu'il est utilisé pour immuniser des souris ou des hamsters dorés par voie générale (intrapéritonéale ou intramusculaire), induit une forte production d'anticorps neutralisants, d'intensité comparable à celles des patients ayant récemment guéri de la Covid-19 symptomatique ou pauci-symptomatique. II est à noter que les vecteurs lentiviraux codant la sous-unité SI seule ou les deux sous-unités S1 et S2 de la protéine Spike, sans le domaine d'ancrage et le segment interne, ne sont pas capables d'induire la production d'anticorps neutralisants, même si ces segments contiennent le domaine RBD. La conformation des épitopes cibles des anticorps neutralisants est donc préservée uniquement lorsque la protéine Spike entière est exprimée, ce qui est concordant avec sa trimérisation. II est important de noter que le LV::S induit également des réponses lymphocytaires $\mathrm{T} \mathrm{CD}^{+}$contre de nombreux épitopes de cette protéine [7].

Pour évaluer l'efficacité protectrice du $L V:: S$ contre le SARS-CoV-2, nous avons d'abord créé un modèle murin dans lequel l'expression du récepteur ACE2 humain par les cellules des voies respiratoires est induite par leur transduction avec un vecteur adénoviral codant ce récepteur. L'épithélium pulmonaire de ces souris devient ainsi très permissif à la réplication du SARS-CoV-2 [7]. Ce modèle offre donc la possibilité d'évaluer in vivo l'efficacité des médicaments ou des candidats vaccins contre la réplication du SARSCoV-2. Dans ce modèle, une vaccination par le LV::S selon un protocole «prime- 


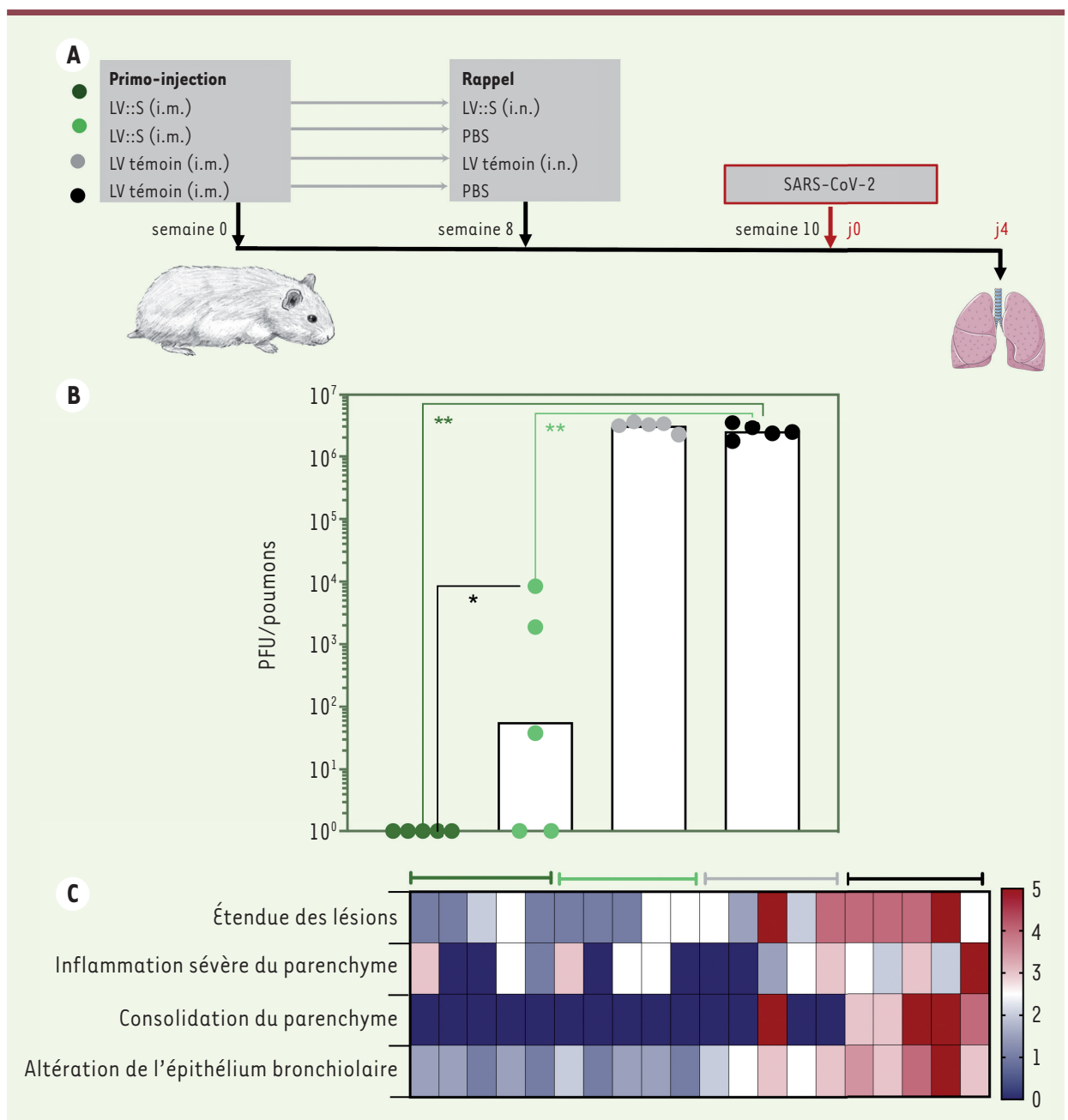

D

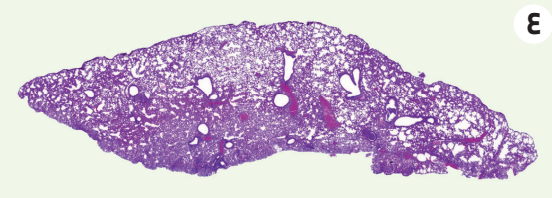

$\varepsilon$

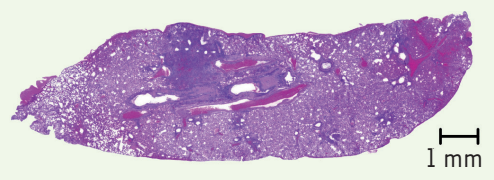

Figure 2. Capacité protectrice de la vaccination par le vecteur lentiviral recombinant $L V:: S$ administré par voie intranasale chez le hamster doré. (A) Protocole de vaccination du hamster doré par LV::S, suivie de l'administration du SARS-CoV-2 par voie intranasale. i.m. = intramusculaire, i.n. = intranasal. PBS désigne une solution saline non immunisante, utilisée comme témoin. Le vecteur $L V$ témoin code un antigène non pertinent pour l'infection. Le code couleur des différents groupes d'animaux testés ( 5 animaux par groupe) est indiqué à gauche de cette figure. (B) Charges virales pulmonaires (plaque-forming units, $P F U$ ) déterminées quatre jours après (j4) après l'inoculation virale (j0). La signification statistique de la différence observée a été évaluée par le test $U$ de Mann-Whitney (* = $p<0,02 ; * \star=p<0,01)$. (c) Analyse histopathologique des poumons, après coloration par hématoxyline et éosine. Heatmap récapitulant les scores histologiques pour chaque paramètre défini et déterminés pour les individus de chaque groupe expérimental. $(D, \varepsilon)$ Coupes du poumon entier de hamsters vaccinés ( $D$ ) et non vaccinés $(\varepsilon)$ au $4^{\mathrm{e}}$ jour postinoculation du SARS-CoV-2. Figure adaptée de [7]. boost $\gg^{2}$ par voie générale confère une protection significative mais partielle contre le SARS-CoV-2 (Figure IA, IB), malgré une activité de séro-neutralisation intense. Cependant, le ciblage de la réponse immunitaire vers les voies respiratoires par un «boost » intranasal aboutit à une protection stérilisante (Figure IA, $1 B$ ) et à une réduction très marquée de l'inflammation et de l'infiltration pulmonaire par des cellules de l'immunité

${ }^{2}$ Le «prime-boost » (amorçage-rappel) est une stratégie de vaccination en plusieurs étapes, qui permet notamment de présenter au système immunitaire un même antigène à I'aide de différents vecteurs. Cette méthode est plus efficace que la stratégie de vaccination classique en une seule étape. innée. Cette protection est corrélée à la présence, dans les poumons, de taux élevés d'Ig(immunoglobulines)A spécifiques de la protéine Spike (Figure 1C) et à une forte activité neutralisante du tissu pulmonaire (Figure 1D) [7]. Cette corrélation établie chez la souris est en accord avec l'observation, chez des patients atteints de la Covid-19, que les IgA sécrétoires contribuent à la neutralisation du SARSCoV-2 avec une plus grande efficacité que les IgG $[9,11]$ $(\rightarrow)$.

$\rightarrow$ Voir la Nouvelle de D. Sterlin et al., $\mathrm{m} / \mathrm{s}$ $n^{\circ} 11$, novembre 2021, page 967

Nous avons également évalué l'efficacité vaccinale de ce vecteur chez le hamster doré, qui est naturellement permissif pour la réplication du SARSCoV-2. Cette espèce animale développe rapidement une maladie pulmonaire majeure après l'inoculation du SARSCoV-2, et constitue un modèle rigoureux d'efficacité vaccinale [10]. Nous avons montré que le LV::S, sous sa forme non intégrative et lorsqu'il est utilisé dans un protocole de «prime» global suivi d'un «boost » intranasal, possède également une très forte efficacité protectrice (Figure 2A, 2B) et empêche la survenue de l'inflammation indésirable (Figure 2C) et des lésions pulmonaires délétères (Figure 2D, 2E) [7]. 
Nous avons donc établi la preuve de principe d'un effet prophylactique efficace de ce candidat vaccin contre le SARS-CoV-2 dans deux modèles précliniques, sur des critères virologiques, immunologiques et histopathologiques. La voie d'administration intranasale permet de recruter des acteurs de l'immunité spécifique, notamment les anticorps de classe IgA et les lymphocytes $T C D 8^{+}$spécifiques de la protéine Spike, dans les muqueuses des voies respiratoires. Cette immunité locale permet de neutraliser directement le virus dès son entrée dans l'organisme hôte, avant qu'il pénètre dans les sites critiques, ce qui est très prometteur pour empêcher la propagation de l'infection dans l'organisme et devrait contribuer à interrompre la chaîne de transmission du virus par voie aérienne. Dans ce cas, ce vaccin protègerait les individus contre la transmission du SARS-CoV-2, et pas seulement contre les signes cliniques de l'infection et les formes sévères de la COVID-19. II faut également noter la nature non-invasive de la voie intranasale d'immunisation, particulièrement adaptée à une vaccination de masse. Nous nous intéressons actuellement à la capacité du LV::S à assurer une protection croisée contre les variants émergents du SARS-CoV-2, dans les poumons et aux autres sites de l'infection. La combinaison d'une forte réponse humorale neutralisante et, surtout, d'une réponse T CD8 ${ }^{+}$polyspécifique est très prometteuse compte tenu de l'importance du rôle protecteur de la composante cellulaire de l'immunisation, et de la conservation quasi complète, dans les différents variants identifiés, des épitopes de la protéine Spike ciblés par la vaccination.

Nous préparons un essai clinique, dont le but est de renforcer l'immunité collective acquise par la vaccination ou par l'infection naturelle, et d'induire ainsi, par une seule instillation intranasale du vaccin, une protection efficace et durable contre les variants du SARS-CoV-2, chez les personnes convalescentes de la Covid-19 ou déjà vaccinées par voie générale. L’intérêt et l'urgence d'une telle approche vaccinale complémentaire dépendra de l'impact de l'émergence des variants du virus sur l'évolution de la pandémie actuelle. $\diamond$

An anti-Covid-19 lentiviral vaccine candidate that can be administered by the nasal route

\section{LIENS D'INTÉR}

$P C$ est le fondateur et directeur scientifique de TheraVectys. $L M$ et $P C$ sont les inventeurs d'un brevet en instance portant sur le potentiel de la vaccination intranasale par le LV::S contre le SARS-CoV-2.

\section{RÉFÉRENCES}

1. Forni G, Mantovani A. Covid-19 commission of Accademia nazionale dei Lincei R. Covid-19 vaccines: where we stand and challenges ahead. Cell Death Differ $2021 ; 28: 626-39$

2. Tiboni M, Casettari L, Illum L. Nasal vaccination against SARS-CoV-2: synergistic or alternative to intramuscular vaccines? Int J Pharm 2021 ; 603 : 120686

3. Ku MW, Charneau P, Majlessi L. Use of lentiviral vectors in vaccination. Expert Rev Vaccines 2021 ; $11: 1-16$

4. Zennou V, Petit C, Guetard D, et al. HIV-1 genome nuclear import is mediated by a central DNA flap. Cell $2000 ; 101: 173-85$.

5. Ku MW, Anna F, Souque P, et al. A single dose of NILVbased vaccine provides rapid and durable protection against Zika virus. Mol Ther $2020 ; 28$ : 1772-82.

6. Ku MW, Authié $P$, Nevo F, et al. Lentiviral vector induces high-quality memory $T$ cells via dendritic cells transduction. Commun Biol 2021 ; 4 : 713-29.

7. Ku MW, Bourgine M, Authie P, et al. Intranasal vaccination with a lentiviral vector protects against SARS-CoV-2 in preclinical animal models. Cell Host Microbe $2021 ; 29$ : 236-49.e6.

8. Walls AC, Park YJ, Tortorici MA, et al. Structure, function, and antigenicity of the SARS-CoV-2 Spike glycoprotein. Cell 2020 ; 181 : 281-92 e6.

9. Sterlin D, Mathian A, Miyara M, et al. IgA dominates the early neutralizing antibody response to SARSCoV-2. Sci Transl Med $2021 ; 13$ : eabd2223.

10. Munoz-Fontela C, Dowling WE, Funnell SGP, et al. Animal models for COVID-19. Nature $2020 ; 586$ : 509-15.

11. Sterlin D, Malaussena A, Gorochov G. Le rôle prépondérant des IgA dans la réponse immunitaire précoce contre le virus SARS-CoV-2. Med Sci (Paris) $2021 ; 37$ (11) : 967-9

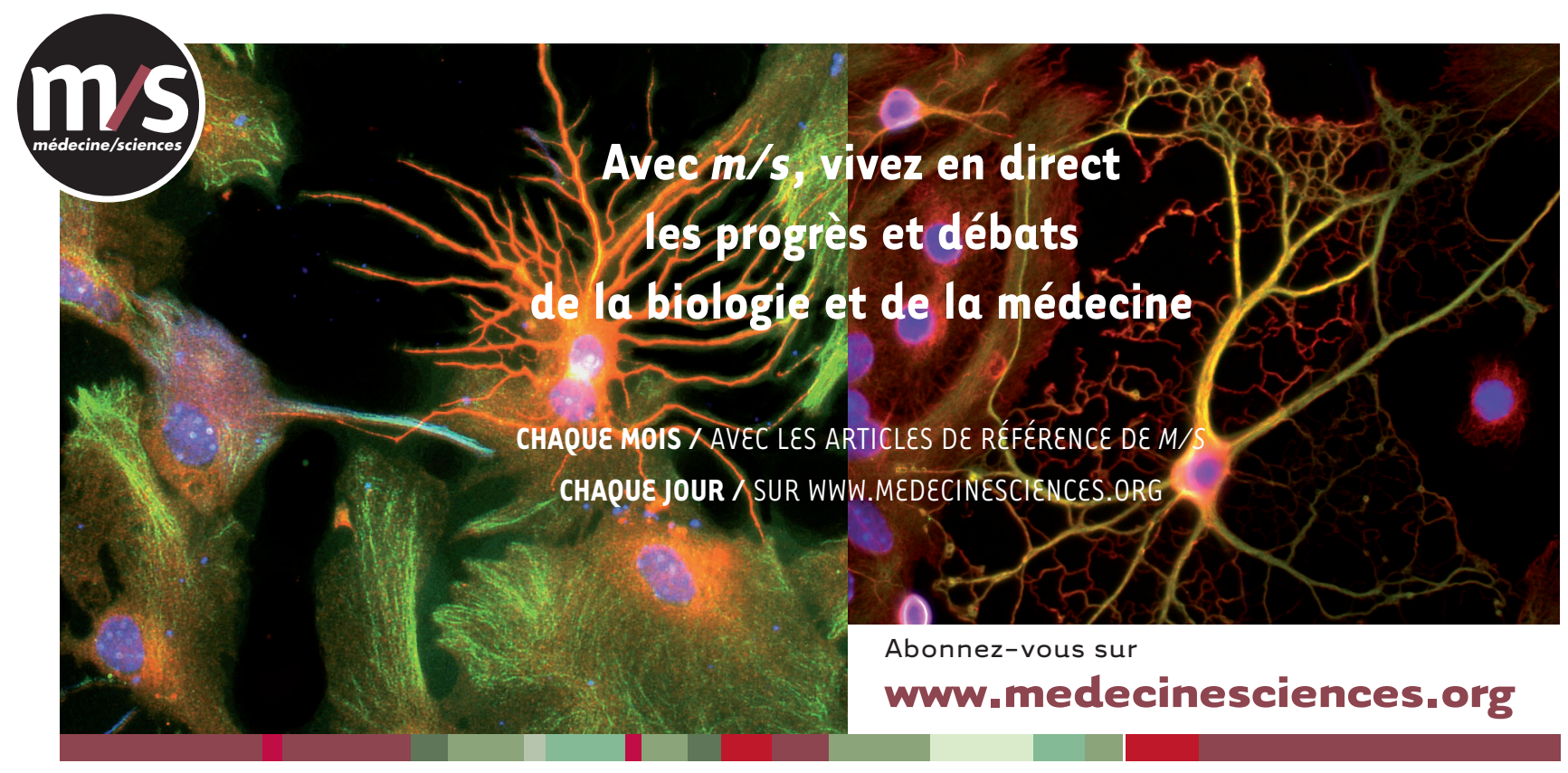

